\title{
Integrated Management of Aphelenchoides besseyi infesting Tuberose in Gangetic Plains of West Bengal
}

\author{
Shimpy Sarkar ${ }^{1 *}$ and Kusal Roy ${ }^{2}$ \\ ${ }^{1}$ Department of Entomology, Lovely Professional University, \\ Phagwara-144411, Punjab, India \\ ${ }^{2}$ Department of Agricultural Entomology, Bidhan Chandra Krishi Viswavidyalaya, Mohanpur, \\ Nadia-741252, West Bengal, India \\ *Corresponding author
}

\section{A B S T R A C T}

\begin{tabular}{|l|}
\hline Ke y w o r d s \\
Aphelenchoides \\
besseyi, Field \\
sanitation, Bulb \\
treatment and \\
Integrated \\
management
\end{tabular}

An experiment was conducted over two years from 2014 to 2016 to find out the suitable management tactics against Aphelenchoides besseyi Christie in tuberose. The experiment was planned in 'Strip Factorial Design'. Three nematode management modules, two susceptible crop cultivars and two spacings were used in the experiment. Management modules were adopted in order to manage the population of $A$. besseyi in tuberose to minimize the infestation for sustainable production of tuberose floral spikes. The IPM module of foliar nematode management comprising of bulb treatment, field sanitation and three rounds of prophylactic spray with nematicides for the 1st and 2 nd year crop in the present experiment kept the $A$. besseyi population significantly lower than the farmers' practice module (bulb treatment + four rounds of prophylactic spray with nematicides for the 1st and 2nd year crop) and untreated control. Hence, adoption of IPM module possibly made the crop to achieve good growth and development as judged by the several attributes (spike length, rachis length, number of nodes/spike, flower yields etc.) measured during the course of experimentation. The maximum return per rupee investment for managing $A$. besseyi in tuberose was observed with the IPM module being, Rs. 17.84. To enhance the flower yield of tuberose, Calcutta Single cultivar can be planted in $30 \mathrm{~cm} \times 25 \mathrm{~cm}$ spacing adopting IPM module of foliar nematode management. Validation and promotion of developed IPM module of foliar nematode management for the farmers raising tuberose in the Gangetic plains of West Bengal need to be done.

\section{Introduction}

Tuberose (Polianthes tuberosa L.) is one of the important tropical bulbous flowering plants grown for production of eternal floral spikes. It is a very popular cut flower in the eastern part of India (West Bengal and Odisha), and also in the northern plains and parts of the south. Its production is constrained mainly by plant parasitic nematodes, insectpests and diseases. Among nematodes, the foliar nematode, Aphelenchoides besseyi Christie has emerged as a serious threat to sustainable tuberose production in West Bengal. Widespread occurrence of foliar nematode infecting rice, tuberose, gladiolus and onion was also reported from West Bengal.

Nematode may survive on bulbs, leaves, scapes, bracts, tepals and anthers but not in the soil (Roy et al., 2010; Roy et al., 2011). Second year onwards tuberose crop suffers most from the infestation of foliar nematode, this may lead to annual yield loss of 30 to $40 \%$ in the 'Calcutta Double' cultivar as against 
$59 \%$ in 'Calcutta Single' cultivar (Khan and Pal, 2001; Pathak and Khan, 2009). International quarantine exists against $A$. besseyi, hence, their presence on cut flowers may limit the potential of flower export. Anhydrobiotic nature of $A$. besseyi creates a barrier against permeability of nematicides into nematode's body, hence making the nematicidal application unfruitful.

Considering the importance of nematodes in the integrated pest management system and increasing concern of environment, adoption of low input plant protection technologies like, summer ploughing, soil solarization, organic manuring, adjustment of dates of sowing/planting, spacing, adoption of resistant varieties, clean cultivation and judicious use of pesticides are gaining significance. Integration of two or more above mentioned methods could be adopted, for managing nematode like, A. besseyi.

In view of its widespread occurrence and increasing importance in the changing agricultural scenario, their integrated management tactics in tuberose was undertaken.

\section{Materials and Methods}

The experiment was conducted over two years from 2014 to 2016 at the Central Research Farm of BCKV, Gayeshpur, Nadia, West Bengal. The farm was located at $22^{\circ} 87^{\prime} \mathrm{N}$ latitude, $88^{\circ} 20^{\prime} \mathrm{E}$ longitude with an elevation of $9.75 \mathrm{~m}$ above mean sea level. The experimental design followed for the experiment was Strip Factorial. Two susceptible crop cultivar were used such as $\mathrm{V}_{1}$ : Calcutta Single, $\mathrm{V}_{2}$ : Calcutta Double and they were planted at two different spacings $S_{1}$ : $30 \mathrm{~cm} \times 20 \mathrm{~cm}$ and $S_{2}: 30 \mathrm{~cm} \times 25 \mathrm{~cm}$, the plot size was $2 \mathrm{~m} \times 1.2 \mathrm{~m}$. Date of sowing of bulbs was 19.04.2014 and the treatments were replicated 3 times.
Treatment Details: $\mathrm{M}_{1}$ : Untreated Control, $\mathrm{M}_{2}$ : Farmers' Practice module: Bulb treatment: Overnight pre-soaking of bulbs followed by dipping in monocrotophos 36 SL @ 720ppm for $4 \mathrm{hrs}$ before planting.

\section{Prophylactic spray}

Four rounds of foliar sprays with monocrotophos 36 SL @ $360 \mathrm{~g}$ a.i./ha at 14 days interval commencing from initiation of infestation for the $1^{\text {st }}$ and $2^{\text {nd }}$ year crop.

$\mathrm{M}_{3}$ : Integrated foliar nematode management module: Bulb treatment: Overnight presoaking of bulbs followed by dipping in cartap hydrochloride 50 SP @1000ppm for 4hrs before planting.

\section{Field sanitation}

Removal of debris from plant and plot periodically (twice in a month) to restrict the migration of nematode and inoculum load.

\section{Prophylactic spray}

First prophylactic spray with cartap hydrochloride 50 SP @ 500g a.i. /ha + Active 80 (adjuvant) @ 5ml/15 litres of water at the time of spike emergence.

Second prophylactic spray with chlorfenapyr 10 SC @ 100g a.i. /ha + Active 80 (adjuvant) @ $5 \mathrm{ml} / 15$ litres of water at 14 days after $1^{\text {st }}$ spray.

Third prophylactic spray with cartap hydrochloride 50 SP @ 500g a.i. /ha + Active 80 (adjuvant) @ 5ml/15 litres of water at 14 days after $2^{\text {nd }}$ spray.

Second year crop received first, second and third spray with above mentioned nematicides and adjuvant at 14 days interval commencing from the spike emergence of tuberose. 
Initial Nematode Population

\begin{tabular}{|l|l|l|l|}
\hline \multirow{2}{*}{ Varieties } & \multicolumn{3}{|l|}{ Population of $\boldsymbol{A}$. besseyi /bulb } \\
\cline { 2 - 4 } & Untreated $\left(\mathbf{M}_{\mathbf{1}}\right)$ & Treated $\left(\mathbf{M}_{\mathbf{2}}\right)$ & Treated $\left(\mathbf{M}_{\mathbf{3}}\right)$ \\
\cline { 2 - 4 } & Before planting & $\begin{array}{l}\text { After bulb treatment } \\
\text { before planting }\end{array}$ & $\begin{array}{l}\text { After bulb treatment } \\
\text { before planting }\end{array}$ \\
\hline Calcutta Single & 3.3 & 1.2 & 0.5 \\
\hline Calcutta Double & 1.4 & 0.7 & 0.2 \\
\hline
\end{tabular}

\section{Observations recorded}

Initial population of A. besseyi / bulb to ascertain presence of nematode, foliar nematode population per $20 \mathrm{~g}$ of plant parts (leaves + flowers) at 14 days interval commencing from appearance symptom, observation on spike length $(\mathrm{cm})$, length of rachis $(\mathrm{cm})$, number of nodes/spike, weight of flowers/spike, spike yield (number/plot and number/ha), weight of flowers $(\mathrm{kg} / \mathrm{plot}$ and $\mathrm{q} / \mathrm{ha}$ ), days to first blooming and $100 \%$ blooming, flower yield in $\mathrm{kg} / \mathrm{plot}$ and $\mathrm{q} / \mathrm{ha}$ and dry yield of bulb and bulb-lets $(\mathrm{kg} / \mathrm{plot}$ and q/ha)

\section{Statistical analysis}

Analysis of variance was worked out following Strip Factorial design for comparison of means (Gomez and Gomez, 1984). Incremental cost benefit ratio for the assessment of the module was also worked out.

\section{Results and Discussion}

Effect of management module, variety and spacing on spike length $(\mathrm{cm})$ of tuberose during 2014-15 and 2015-16

The effect of management module, variety and spacing on spike length of tuberose during 2014-15 and 2015-16 has been presented in the table 1. There was no significant difference in spike length among the management modules during the $1^{\text {st }}$ year but during $2^{\text {nd }}$ year a significant variation in spike length among management modules was noticed and the maximum length was observed in the $\mathrm{M}_{3}$ (IPM module) being, 92.1 $\mathrm{cm}$. Pooled data also revealed significant difference in spike length among management modules with the maximum being, $91.0 \mathrm{~cm}$ was recorded in the $\mathrm{M}_{3}$ i.e. IPM module (Table 1). Significant difference in spike length among varieties was observed only during $1^{\text {st }}$ year, where Calcutta Single had maximum spike length $(94.6 \mathrm{~cm})$. Nonsignificant effect of variety on the spike length of tuberose was noticed in the $2^{\text {nd }}$ year. Pooled data revealed significant influence of variety on the spike length. Based on pooled data highest spike length was noted with the Calcutta Single variety being, $89.9 \mathrm{~cm}$ (Table 1). The spacings adopted to raise the tuberose crop did not reveal significant influence on the spike length (Table 1). Usually, spike length was observed maximum when Calcutta Single variety was planted in wide spacing and adopted IPM module.

Effect of management module, variety and spacing on rachis length $(\mathrm{cm})$ of tuberose during 2014-15 and 2015-16

The effect of management module, variety and spacing on rachis length of tuberose during 2014-15 and 2015-16 has been presented in the table 2. Significant difference in rachis length among the management modules was observed in both the years with the maximum in the IPM module $\left(\mathrm{M}_{3}\right)$. Considering pooled data, maximum length of tuberose rachis was recorded in the $\mathrm{M}_{3}$ i.e. IPM module being, $36.7 \mathrm{~cm}$ (Table 2). Tuberose variety also had a significant influence on the rachis length 
during study period. Calcutta Double was found superior to Calcutta Single in achieving rachis length. Significant maximum rachis length in both the years was observed with the Calcutta Double variety being, $33.6 \mathrm{~cm}$ and $28.7 \mathrm{~cm}$, respectively. Likewise, pooled data also revealed similar trend with regard to the rachis length.

Spacing revealed significant impact on the rachis length only during second year of the study. Wide spaced crop had comparatively more rachis length than the narrow spaced crop (Table 2).

Effect of management module, variety and spacing on number of nodes/spike of tuberose during 2014-15 and 2015-16

Node is an important botanical part of tuberose spike. Here, flower buds originate from the node. More number of nodes means more sites to accommodate flowers. Hence, observation on number of nodes/spike has the value to judge the performance of treatments on the tuberose crop.

The effect of management module, variety and spacing on number of nodes/spike of tuberose during 2014-15 and 2015-16 has been presented here in the table 3. Foliar nematode management module had non- significant impact on the production of nodes/spike in the $1^{\text {st }}$ year crop. In contrast, the $2^{\text {nd }}$ year crop had significant difference in the number of nodes/spike between management modules. The highest number of nodes/spike was always recorded in the IPM module. Mean number of nodes/spike in the IPM module was 14.0 (pooled).

Unlike nematode management module, variety too had an influence on the production of number of nodes/spike. Calcutta Double variety produced more number of nodes/spike than the Calcutta Single variety. Mean number of nodes/spike in the Calcutta Double variety was 14.0 (pooled).
Spacing didn't reveal any effect on number of nodes/spike.

Effect of foliar nematode management module, variety and spacing on number of flowers/spike of tuberose during 2014-15 and 2015-16

Effect of foliar nematode management module, variety and spacing on production of flowers/spike in tuberose during 2014-15 and 2015-16 has been presented below in the table 4. Production of flowers/spike was maximum in the IPM module as confirmed by the pooled result of two years study. Number of flowers/spike was 28.7 (pooled).

Variety exhibited significant influence on the production of flowers/spike during the period of experimentation. In reference to the mentioned yield attribute, the variety Calcutta Double was superior to the Calcutta Single. Flower yield (number) of Calcutta Double variety was $28.6 /$ spike (pooled).

Spacing showed significant influence on flower yield only in the $1^{\text {st }}$ year crop, but the ultimate effect of spacing on yield of flowers (number/spike) was non-significant. In a general perspective, irrespective of the variety and the nematode management module the wide spaced crop bore more flowers/spike (Table 4).

Effect of management module, spacing and variety on days to $1^{\text {st }}$ and $100 \%$ blooming of tuberose flower

Effect of management module, spacing and variety on days to $1^{\text {st }}$ blooming and $100 \%$ blooming of tuberose flower has been presented in the table 5. The foliar nematode management modules and spacings in the experiment had non-significant impact on the number of days required to attain $1^{\text {st }}$ as well as $100 \%$ blooming of tuberose flower. Data showed comparatively lesser requirement of 
time to attain $1^{\text {st }}$ and $100 \%$ blooming of flowers in the plots received IPM module or where bulbs were planted in narrow spacing $\left(\mathrm{S}_{1}\right)$.

The varieties played significant role on the blooming of flowers as observed from the data (Table 5). Calcutta Single took longer time to start its blooming i.e. 192.6 days after planting while, $100 \%$ blooming of spikes in the variety was observed at 435.1 days after planting. In contrast, the Calcutta Double took only 117.2 days to start blooming and $100 \%$ blooming was attained in 415.0 days after planting of bulbs.

Desai et al., (2016) reported that spacing had non-significant effect on flowering of tuberose cv. Shringar. Mishra et al., (2000) observed that tuberose cultivars (Single and Double) with closer spacing took longer time to produce spikes than wide-spaced plants. Malam et al., (2010) reported that days to spike emergence and days to first flower opening decreased with increase in spacing of tuberose cv. Double. Ratoon crop flowers early as compared to the first year crop. In the present study, early emergence of spike and flowers in narrow-spaced tuberose crop might be due to reduction of growth that possibly directed the plant towards the reproductive phase earlier than the plant that acquired more vegetative growth at wider spaces. Similar observations on the mentioned aspect were earlier cited by several workers (Mohanty et al., 2002; Ahmed et al., 2010, Sheoran et al., 2015).

Effect of management module, variety and spacing on weight of flowers (g/spike) and flower yield of tuberose during 2014-15 and 2015-16

Effect of management module, variety and spacing on weight of flowers (g/spike) of tuberose during 2014-15 and 2015-16 has been presented below in the table 6 .
Significant difference in the weight of flowers (g/spike) among foliar nematode management module was observed during $2^{\text {nd }}$ year. The ultimate effect of management module on the weight of flowers was significant. The maximum weight of flowers was recorded in the IPM module $\left(\mathrm{M}_{3}\right)$ being, $45.6 \mathrm{~g} / \mathrm{spike}$ (pooled).

Calcutta Double variety has significantly maximum weight of flowers being, 63.4 and $58.9 \mathrm{~g} /$ spike in the $1^{\text {st }}$ and $2^{\text {nd }}$ year, respectively. Pooled data also showed similar influence of variety on the weight of flowers/spike (Table 6).

Spacing had significant effect on weight of flowers/spike only during $1^{\text {st }}$ year where widespaced crop gave more weight of flowers (g/spike) than the narrow spaced crop (Table $6)$.

Impact of management module, variety and spacing on the flower yield $(\mathrm{kg} / \mathrm{plot}$ and $\mathrm{q} / \mathrm{ha})$ of tuberose was significant in both the years (Table 7 and 8). Flower yield per plot as well as per ha was observed significantly maximum in the IPM module being, $2.6 \mathrm{~kg}$ and $109.1 \mathrm{q}$, respectively (pooled).

Effect of spacing and variety on the yield of flowers $(\mathrm{kg} / \mathrm{plot}$ or $\mathrm{q} / \mathrm{ha})$ was significant in both the years (Table 7 and 8). Flower yield was more in the wider spacing $(30 \mathrm{~cm} \times 25 \mathrm{~cm})$ than the narrower spacing $(30 \mathrm{~cm} \times 20 \mathrm{~cm})$.

Loose flower yield of tuberose was maximum in the wide spaced crop being, 85.5 and 88.2 $\mathrm{q} /$ ha in the $1^{\text {st }}$ and $2^{\text {nd }}$ year, respectively (Table 8 ). Flower yield of tuberose was observed maximum in the Calcutta Single variety being, 88.7 and $91.1 \mathrm{q} / \mathrm{ha}$ in the $1^{\text {st }}$ and $2^{\text {nd }}$ year, respectively (Table 8 ). In spite of having more weight of flowers/spike, Calcutta Double cultivar couldn't produce more flower yield $(\mathrm{kg} / \mathrm{plot}$ and $\mathrm{q} / \mathrm{ha})$. Flower yield depends more importantly on the number of floral spike 
production per unit area. Production of tuberose spike/unit area was more in the Calcutta Single than the Calcutta Double. Thus, despite less weight of flowers/spike, the flower yield in Calcutta Single cultivar was more than the Calcutta Double cultivar. Nematode management module and spacing posed significant impact on the flower yield ( $\mathrm{kg} / \mathrm{plot}$ and $\mathrm{q} / \mathrm{ha}$ ) of tuberose as confirmed by the pooled result (Table 7, 8). Here, adoption of IPM module along with wide spacing fetched more flower yield.

Effect of management module, spacing and variety on bulb yield of tuberose

Bulb yield ( $\mathrm{kg} /$ plot and $\mathrm{q} / \mathrm{ha}$ ) of tuberose varied significantly due to adoption of foliar nematode management modules. Irrespective of spacings and varieties, the IPM module played an important role in achieving maximum bulb yield of tuberose being, 10.9 $\mathrm{kg} /$ plot and 454.7q/ha (Table 9). Among varieties used in the experiment, the Calcutta Double yielded maximum bulb which was 8.9 $\mathrm{kg} / \mathrm{plot}$ and $369.8 \mathrm{q} / \mathrm{ha}$ (Table 9). In the present study, spacing had non-significant impact on the bulb production of tuberose.

Effect of management module, spacing and variety on spike yield of tuberose during 2014-15 and 2015-16

Adoption of foliar nematode (A. besseyi) management module, variety and spacing played an important role in influencing the spike yield (number/plot and number/ha) of tuberose. Maximum yield (number) of tuberose spike was obtained from the IPM module $\left(\mathrm{M}_{3}\right)$ being, 84.7/2.4 $\mathrm{m}^{2}$ plot and 3,52,777.8/ha during 2014-15 (Table 10). Second year and pooled result showed nonsignificant effect of nematode management module on the spike yield but the $\mathrm{M}_{3}$ yielded more number of spikes per plot as well as per ha.

Table.1 Effect of management module, variety and spacing on spike length of tuberose during 2014-15 and 2015-16

\begin{tabular}{|l|c|c|c|}
\hline \multirow{2}{*}{ Treatments } & \multicolumn{3}{|c|}{ Spike length $(\mathbf{c m})$} \\
\cline { 2 - 4 } & $\mathbf{2 0 1 4 - 1 5}$ & $\mathbf{2 0 1 5 - 1 6}$ & Pooled \\
\hline $\mathbf{M}_{\mathbf{1}}$ & 87.1 & 80.2 & 83.6 \\
\hline $\mathbf{M}_{\mathbf{2}}$ & 86.8 & 85.7 & 86.3 \\
\hline $\mathbf{M}_{\mathbf{3}}$ & 89.9 & 92.1 & 91.0 \\
\hline SEm $( \pm)$ & $\mathbf{1 . 3 2}$ & $\mathbf{1 . 5 7}$ & $\mathbf{1 . 1 7}$ \\
\hline LSD $(\mathbf{0 . 0 5})$ & $\mathbf{N S}$ & $\mathbf{4 . 8 4}$ & $\mathbf{3 . 4 5}$ \\
\hline $\mathbf{V}_{\mathbf{1}}:$ Calcutta Single & 94.6 & 85.3 & 89.9 \\
\hline $\mathbf{V}_{\mathbf{2}}:$ Calcutta Double & 81.3 & 86.7 & 84.0 \\
\hline SEm $(\mathbf{\pm})$ & $\mathbf{0 . 7 0}$ & $\mathbf{0 . 7 3}$ & $\mathbf{0 . 8 5}$ \\
\hline $\mathbf{L S D}(\mathbf{0 . 0 5})$ & $\mathbf{2 . 1 6}$ & $\mathbf{N S}$ & $\mathbf{2 . 5 1}$ \\
\hline $\mathbf{S}_{\mathbf{1}}:(\mathbf{3 0} \times \mathbf{2 0}) \mathbf{c m}$ & 87.0 & 85.8 & 86.4 \\
\hline $\left.\mathbf{S}_{\mathbf{2}}: \mathbf{3 0} \times \mathbf{2 5}\right) \mathbf{c m}$ & 88.9 & 86.2 & 87.5 \\
\hline SEm $( \pm)$ & $\mathbf{1 . 6 2}$ & $\mathbf{1 . 2 9}$ & $\mathbf{1 . 1 7}$ \\
\hline $\mathbf{L S D}(\mathbf{0 . 0 5})$ & $\mathbf{N S}$ & $\mathbf{N S}$ & $\mathbf{N S}$ \\
\hline
\end{tabular}


Table.2 Effect of management module, variety and spacing on rachis length of tuberose during 2014-15 and 2015-16

\begin{tabular}{|c|c|c|c|}
\hline \multirow[t]{2}{*}{ Treatments } & \multicolumn{3}{|c|}{ Rachis length (cm) } \\
\hline & 2014-15 & 2015-16 & Pooled \\
\hline $\mathbf{M}_{1}$ & 25.6 & 16.6 & 11.5 \\
\hline $\mathbf{M}_{2}$ & 25.4 & 25.9 & 21.4 \\
\hline $\mathbf{M}_{3}$ & 27.4 & 38.6 & 36.7 \\
\hline $\operatorname{SEm}( \pm)$ & 0.40 & 0.88 & 0.55 \\
\hline LSD (0.05) & 1.23 & 2.71 & 1.62 \\
\hline $\mathrm{V}_{1}:$ Calcutta Single & 18.7 & 25.3 & 22.0 \\
\hline $\mathrm{V}_{2}:$ Calcutta Double & 33.6 & 28.7 & 31.1 \\
\hline $\operatorname{SEm}( \pm)$ & 0.39 & 0.55 & 0.26 \\
\hline LSD (0.05) & 1.20 & 1.69 & 0.77 \\
\hline $\mathrm{S}_{1}:(30 \times 20) \mathrm{cm}$ & 25.4 & 26.4 & 25.9 \\
\hline$S_{2}:(30 \times 25) \mathrm{cm}$ & 26.8 & 27.6 & 27.2 \\
\hline $\operatorname{SEm}( \pm)$ & 0.43 & 0.12 & 0.39 \\
\hline LSD (0.05) & NS & 0.37 & NS \\
\hline
\end{tabular}

Note: $\mathrm{M}_{1}=$ Untreated control, $\mathrm{M}_{2}=$ Farmer's practice module and $\mathrm{M}_{3}=$ Integrated pest management module

Table.3 Effect of management module, variety and spacing on number of nodes/spike of tuberose during 2014-15 and 2015-16

\begin{tabular}{|c|c|c|c|}
\hline \multirow[t]{2}{*}{ Treatments } & \multicolumn{3}{|c|}{ Number of nodes/spike } \\
\hline & 2014-15 & 2015-16 & Pooled \\
\hline $\mathbf{M}_{1}$ & 10 & 9 & 10 \\
\hline $\mathbf{M}_{2}$ & 11 & 14 & 12 \\
\hline $\mathbf{M}_{3}$ & 11 & 17 & 14 \\
\hline $\operatorname{SEm}( \pm)$ & 0.31 & 0.26 & 0.23 \\
\hline LSD (0.05) & NS & 0.80 & 0.68 \\
\hline $\mathrm{V}_{1}$ :Calcutta Single & 8 & 12 & 10 \\
\hline $\mathrm{V}_{2}$ :Calcutta Double & 13 & 15 & 14 \\
\hline SEm $( \pm)$ & 0.12 & 0.24 & 0.12 \\
\hline LSD (0.05) & 0.37 & 0.74 & 0.35 \\
\hline$S_{1}:(30 \times 20) \mathrm{cm}$ & 11 & 13 & 12 \\
\hline$S_{2}:(30 \times 25) \mathrm{cm}$ & 11 & 14 & 12 \\
\hline $\operatorname{SEm}( \pm)$ & 0.17 & 0.18 & 0.16 \\
\hline LSD (0.05) & NS & NS & NS \\
\hline
\end{tabular}


Table.4 Effect of foliar nematode management module, variety and spacing on number of flowers/spike of tuberose during 2014-15 and 2015-16

\begin{tabular}{|l|c|c|c|}
\hline \multirow{2}{*}{ Treatments } & \multicolumn{3}{|c|}{ Number of flowers/spike } \\
\cline { 2 - 4 } & $\mathbf{2 0 1 4 - 1 5}$ & $\mathbf{2 0 1 5 - 1 6}$ & Pooled \\
\hline $\mathbf{M}_{\mathbf{1}}$ & 22.1 & 18.9 & 20.5 \\
\hline $\mathbf{M}_{\mathbf{2}}$ & 21.8 & 28.0 & 24.9 \\
\hline $\mathbf{M}_{\mathbf{3}}$ & 23.5 & 33.9 & 28.7 \\
\hline SEm $(\mathbf{\pm})$ & $\mathbf{0 . 6 4}$ & $\mathbf{0 . 5 3}$ & $\mathbf{0 . 4 6}$ \\
\hline LSD $(\mathbf{0 . 0 5})$ & $\mathbf{N S}$ & $\mathbf{1 . 6 3}$ & $\mathbf{1 . 3 6}$ \\
\hline $\mathbf{V}_{\mathbf{1}}:$ Calcutta Single & 17.0 & 24.6 & 20.8 \\
\hline $\mathbf{V}_{\mathbf{2}}:$ Calcutta Double & 28.0 & 29.2 & 28.6 \\
\hline SEm $(\mathbf{\pm})$ & $\mathbf{0 . 3 7}$ & $\mathbf{0 . 4 8}$ & $\mathbf{0 . 2 3}$ \\
\hline LSD $(\mathbf{0 . 0 5})$ & $\mathbf{1 . 1 4}$ & $\mathbf{1 . 4 8}$ & $\mathbf{0 . 6 8}$ \\
\hline $\mathbf{S}_{\mathbf{1}}:(\mathbf{3 0} \times \mathbf{2 0}) \mathbf{c m}$ & 22.0 & 26.5 & 24.3 \\
\hline $\left.\mathbf{S}_{\mathbf{2}}: \mathbf{( 3 0} \times \mathbf{2 5}\right) \mathbf{c m}$ & 22.9 & 27.4 & 25.1 \\
\hline SEm $(\mathbf{\pm})$ & $\mathbf{0 . 1 5}$ & $\mathbf{0 . 3 5}$ & $\mathbf{0 . 3 3}$ \\
\hline LSD $(\mathbf{0 . 0 5})$ & $\mathbf{0 . 4 6}$ & $\mathbf{N S}$ & $\mathbf{N S}$ \\
\hline
\end{tabular}

Table.5 Effect of management module, spacing and variety on days to 1 st and $100 \%$ blooming of tuberose flower (mean of two crop seasons)

\begin{tabular}{|l|c|c|}
\hline Treatments & Days to first blooming & Days to $\mathbf{1 0 0 \%}$ blooming \\
\hline $\mathbf{M}_{\mathbf{1}}$ & 160.5 & 428.0 \\
\hline $\mathbf{M}_{\mathbf{2}}$ & 152.2 & 425.8 \\
\hline $\mathbf{M}_{\mathbf{3}}$ & 151.9 & 421.3 \\
\hline SEm $( \pm)$ & $\mathbf{1 2 . 9 1}$ & $\mathbf{2 . 5 8}$ \\
\hline $\mathbf{L S D}(\mathbf{0 . 0 5})$ & $\mathbf{N S}$ & $\mathbf{N S}$ \\
\hline $\mathbf{V}_{\mathbf{1}}:$ Calcutta Single & 192.6 & 435.1 \\
\hline $\mathbf{V}_{\mathbf{2}}:$ Calcutta Double & 117.2 & 415.0 \\
\hline $\mathbf{S E m}(\mathbf{\pm})$ & $\mathbf{8 . 6 2}$ & $\mathbf{1 . 7 9}$ \\
\hline $\mathbf{L S D}(\mathbf{0 . 0 5})$ & $\mathbf{2 6 . 5 6}$ & $\mathbf{5 . 5 2}$ \\
\hline $\left.\mathbf{S}_{\mathbf{1}} \mathbf{( 3 0} \times \mathbf{2 0}\right) \mathbf{c m}$ & 146.1 & 423.6 \\
\hline $\left.\mathbf{S}_{\mathbf{2}} \mathbf{( \mathbf { 3 0 }} \times \mathbf{2 5}\right) \mathbf{c m}$ & 163.7 & 426.6 \\
\hline $\mathbf{S E m}(\mathbf{(})$ & $\mathbf{1 1 . 8 1}$ & $\mathbf{2 . 0 0}$ \\
\hline $\mathbf{L S D}(\mathbf{0 . 0 5})$ & $\mathbf{N S}$ & $\mathbf{N S}$ \\
\hline
\end{tabular}

Note: $\mathrm{M}_{1}=$ Untreated control, $\mathrm{M}_{2}=$ Farmer's practice module and $\mathrm{M}_{3}=$ Integrated pest management module 
Table.6 Effect of management module, variety and spacing on weight of flowers (g/spike) of tuberose during 2014-15 and 2015-16

\begin{tabular}{|c|c|c|c|}
\hline \multirow[t]{2}{*}{ Treatments } & \multicolumn{3}{|c|}{ Weight of flowers (g/spike) } \\
\hline & 2014-15 & 2015-16 & Pooled \\
\hline $\mathbf{M}_{1}$ & 38.5 & 28.8 & 33.7 \\
\hline $\mathbf{M}_{2}$ & 37.7 & 42.3 & 40.0 \\
\hline $\mathbf{M}_{3}$ & 41.3 & 49.9 & 45.6 \\
\hline $\operatorname{SEm}( \pm)$ & 1.09 & 1.15 & 0.93 \\
\hline LSD (0.05) & NS & 3.54 & 2.74 \\
\hline $\mathrm{V}_{1}$ : Calcutta Single & 14.9 & 21.8 & 18.3 \\
\hline $\mathrm{V}_{2}$ : Calcutta Double & 63.4 & 58.9 & 61.2 \\
\hline $\operatorname{SEm}( \pm)$ & 0.73 & 0.81 & 0.48 \\
\hline LSD (0.05) & 2.25 & 2.50 & 1.42 \\
\hline$S_{1}:(30 \times 20) \mathrm{cm}$ & 37.9 & 39.9 & 38.9 \\
\hline$S_{2}:(30 \times 25) \mathrm{cm}$ & 40.4 & 40.8 & 40.6 \\
\hline $\operatorname{SEm}( \pm)$ & 0.16 & 0.86 & 0.65 \\
\hline LSD (0.05) & 1.48 & NS & NS \\
\hline
\end{tabular}

Table.7 Effect of management module, variety and spacing on flower yield (kg/plot) of tuberose during 2014-15 and 2015-16

\begin{tabular}{|c|c|c|c|}
\hline \multirow[t]{2}{*}{ Treatments } & \multicolumn{3}{|c|}{ Flower yield (kg/plot) } \\
\hline & 2014-15 & 2015-16 & Pooled \\
\hline $\mathbf{M}_{1}$ & 1.5 & 1.0 & 1.2 \\
\hline $\mathbf{M}_{2}$ & 1.8 & 1.9 & 1.9 \\
\hline $\mathbf{M}_{3}$ & 2.4 & 2.9 & 2.6 \\
\hline $\operatorname{SEm}( \pm)$ & 0.17 & 0.18 & 0.23 \\
\hline LSD (0.05) & 0.52 & 0.55 & 0.68 \\
\hline $\mathrm{V}_{1}:$ Calcutta Single & 1.6 & 1.7 & 1.9 \\
\hline $\mathrm{V}_{2}$ : Calcutta Double & 2.1 & 2.2 & 1.9 \\
\hline $\operatorname{SEm}( \pm)$ & 0.14 & 0.10 & 0.02 \\
\hline LSD (0.05) & 0.43 & 0.31 & NS \\
\hline$S_{1}:(30 \times 20) \mathrm{cm}$ & 1.7 & 1.8 & 1.7 \\
\hline$S_{2}:(30 \times 25) \mathrm{cm}$ & 2.1 & 1.9 & 2.1 \\
\hline SEm $( \pm)$ & 0.03 & 0.05 & 0.19 \\
\hline LSD (0.05) & 0.09 & 0.15 & NS \\
\hline
\end{tabular}


Table.8 Effect of management module, variety and spacing on flower yield (q/ha) of tuberose during 2014-15 and 2015-16

\begin{tabular}{|c|c|c|c|}
\hline \multirow[t]{2}{*}{ Treatments } & \multicolumn{3}{|c|}{ Flower yield (q/ha) } \\
\hline & 2014-15 & 2015-16 & Pooled \\
\hline $\mathbf{M}_{1}$ & 61.4 & 43.0 & 52.2 \\
\hline $\mathbf{M}_{2}$ & 73.8 & 80.5 & 77.1 \\
\hline $\mathbf{M}_{3}$ & 98.1 & 120.1 & 109.1 \\
\hline $\operatorname{SEm}( \pm)$ & 7.15 & 7.57 & 9.60 \\
\hline LSD (0.05) & 22.03 & 23.33 & 28.32 \\
\hline $\mathrm{V}_{1}:$ Calcutta Single & 88.7 & 91.1 & 79.9 \\
\hline$V_{2}:$ Calcutta Double & 66.8 & 71.2 & 79.0 \\
\hline $\operatorname{SEm}( \pm)$ & 5.67 & 4.04 & 1.08 \\
\hline LSD (0.05) & 17.47 & 12.45 & NS \\
\hline$S_{1}:(30 \times 20) \mathrm{cm}$ & 69.9 & 74.2 & 72.0 \\
\hline$S_{2}:(30 \times 25) \mathrm{cm}$ & 85.5 & 88.2 & 86.9 \\
\hline $\operatorname{SEm}( \pm)$ & 1.06 & 2.43 & 7.86 \\
\hline LSD (0.05) & 3.27 & 7.49 & NS \\
\hline
\end{tabular}

Note: $\mathrm{M}_{1}=$ Untreated control, $\mathrm{M}_{2}=$ Farmer's practice module and $\mathrm{M}_{3}=$ Integrated pest management module

Table.9 Effect of management module, spacing and variety on bulb yield of tuberose

\begin{tabular}{|c|c|c|}
\hline Treatments & 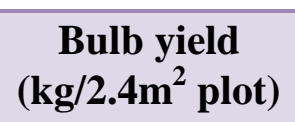 & $\begin{array}{c}\text { Bulb yield } \\
\text { (q/ha) }\end{array}$ \\
\hline $\mathbf{M}_{1}$ & 5.3 & 221.1 \\
\hline $\mathbf{M}_{2}$ & 7.8 & 334.2 \\
\hline $\mathbf{M}_{3}$ & 10.9 & 454.7 \\
\hline $\operatorname{SEm}( \pm)$ & 0.31 & 12.91 \\
\hline LSD (0.05) & 0.96 & 39.78 \\
\hline $\mathrm{V}_{1}$ : Calcutta Single & 7.3 & 303.5 \\
\hline $\mathrm{V}_{2}$ : Calcutta Double & 8.9 & 369.8 \\
\hline $\operatorname{SEm}( \pm)$ & 0.34 & 14.47 \\
\hline LSD (0.05) & 1.05 & 44.59 \\
\hline$S_{1}:(30 \times 20) \mathrm{cm}$ & 8.1 & 337.1 \\
\hline$S_{2}:(30 \times 25) \mathrm{cm}$ & 8.1 & 336.2 \\
\hline $\operatorname{SEm}( \pm)$ & 0.16 & 6.81 \\
\hline LSD (0.05) & NS & NS \\
\hline
\end{tabular}


Table.10 Effect of management module, spacing and variety on spike yield (number/plot) of tuberose during 2014-15 and 2015-16

\begin{tabular}{|c|c|c|c|}
\hline \multirow[t]{2}{*}{ Treatments } & \multicolumn{3}{|c|}{ Spike yield (number $/ 2.4 \mathrm{~m}^{2}$ plot) } \\
\hline & 2014-15 & 2015-16 & Pooled \\
\hline $\mathbf{M}_{1}$ & 55.4 & 49.3 & 52.4 \\
\hline $\mathbf{M}_{2}$ & 69.1 & 60.6 & 64.8 \\
\hline $\mathbf{M}_{3}$ & 84.7 & 76.2 & 80.5 \\
\hline $\operatorname{SEm}( \pm)$ & 5.41 & 5.97 & 6.70 \\
\hline LSD (0.05) & 16.67 & NS & NS \\
\hline$V_{1}$ : Calcutta Single & 106.5 & 96.0 & 101.3 \\
\hline $\mathrm{V}_{2}$ : Calcutta Double & 32.9 & 28.1 & 30.5 \\
\hline $\operatorname{SEm}( \pm)$ & 4.21 & 3.75 & 3.15 \\
\hline LSD (0.05) & 12.97 & 11.55 & 9.29 \\
\hline$S_{1}:(30 \times 20) \mathrm{cm}$ & 64.8 & 59.0 & 61.9 \\
\hline$S_{2}:(30 \times 25) \mathrm{cm}$ & 74.6 & 65.1 & 69.8 \\
\hline $\operatorname{SEm}( \pm)$ & 0.93 & 0.91 & 5.11 \\
\hline LSD (0.05) & 2.87 & 2.80 & NS \\
\hline
\end{tabular}

Table.11 Effect of management module, spacing and variety on spike yield (number/ha) of tuberose during 2014-15 and 2015-16

\begin{tabular}{|c|c|c|c|}
\hline \multirow[t]{2}{*}{ Treatments } & \multicolumn{3}{|c|}{ Spike yield (number/ha) } \\
\hline & 2014-15 & 2015-16 & Pooled \\
\hline $\mathbf{M}_{1}$ & 230903 & 205556 & 218229 \\
\hline $\mathbf{M}_{2}$ & 287847 & 252431 & 270139 \\
\hline $\mathbf{M}_{3}$ & 352778 & 317361 & 335070 \\
\hline $\operatorname{SEm}( \pm)$ & 22527.59 & 24125.83 & 27928.01 \\
\hline $\operatorname{LSD}(0.05)$ & 69414.41 & NS & NS \\
\hline $\mathrm{V}_{1}$ : Calcutta Single & 443750 & 400000 & 421875 \\
\hline $\mathrm{V}_{2}$ : Calcutta Double & 137269 & 116898 & 127083 \\
\hline $\operatorname{SEm}( \pm)$ & 17535.41 & 15604.84 & 13137.89 \\
\hline $\operatorname{LSD}(0.05)$ & 54031.98 & 48083.30 & 38756.75 \\
\hline $\mathrm{S}_{1}:(30 \times 20) \mathrm{cm}$ & 270139 & 245833 & 257986 \\
\hline$S_{2}:(30 \times 25) \mathrm{cm}$ & 310880 & 271065 & 290972 \\
\hline $\operatorname{SEm}( \pm)$ & 3870.00 & 3807.13 & 21307.78 \\
\hline $\operatorname{LSD}(0.05)$ & 11924.66 & 11730.93 & NS \\
\hline
\end{tabular}


Table.12 Effect of management module, variety and spacing on population of $A$. besseyi in tuberose at different days after spray during 2014-15

\begin{tabular}{|c|c|c|c|c|c|c|c|}
\hline \multirow[t]{3}{*}{ Treatments } & \multicolumn{7}{|c|}{ Population of $A$. besseyi / $20 \mathrm{~g}$ of plant parts } \\
\hline & \multicolumn{7}{|c|}{ 2014-15 } \\
\hline & $\begin{array}{l}\text { Before } \\
\text { spray }\end{array}$ & $\begin{array}{c}7 \text { days } \\
{\text { after } 1^{\text {st }}}_{\text {spray }}\end{array}$ & $\begin{array}{c}14 \text { days } \\
\text { after 1st } \\
\text { spray }\end{array}$ & $\begin{array}{c}7 \text { days } \\
\text { after } 2^{\text {nd }} \\
\text { spray }\end{array}$ & $\begin{array}{l}14 \text { days } \\
\text { after } 2^{\text {nd }} \\
\text { spray }\end{array}$ & $\begin{array}{c}7 \text { days } \\
\text { after } 3^{\text {rd }} \\
\text { spray }\end{array}$ & $\begin{array}{c}14 \text { days } \\
\text { after } 3^{\text {rd }} \\
\text { spray }\end{array}$ \\
\hline $\mathbf{M}_{1}$ & $\begin{array}{c}10.6 \\
(141.0)\end{array}$ & $\begin{array}{c}10.6 \\
(113.8)\end{array}$ & $\begin{array}{c}10.8 \\
(115.8)\end{array}$ & $\begin{array}{c}11.0 \\
(120.9)\end{array}$ & $\begin{array}{c}10.7 \\
(114.5)\end{array}$ & $\begin{array}{c}9.7 \\
(94.6)\end{array}$ & $\begin{array}{c}9.4 \\
(87.4)\end{array}$ \\
\hline $\mathbf{M}_{2}$ & $\begin{array}{c}10.0 \\
(100.3)\end{array}$ & $\begin{array}{c}9.2 \\
(88.2)\end{array}$ & $\begin{array}{c}8.7 \\
(75.9)\end{array}$ & $\begin{array}{c}8.9 \\
(78.8)\end{array}$ & $\begin{array}{c}7.2 \\
(52.7)\end{array}$ & $\begin{array}{c}4.9 \\
(24.3)\end{array}$ & $\begin{array}{c}7.0 \\
(49.3)\end{array}$ \\
\hline $\mathbf{M}_{3}$ & $\begin{array}{c}8.7 \\
(79.5)\end{array}$ & $\begin{array}{c}9.2 \\
(83.9)\end{array}$ & $\begin{array}{c}8.3 \\
(68.9)\end{array}$ & $\begin{array}{c}7.7 \\
(59.8)\end{array}$ & $\begin{array}{c}4.0 \\
(15.9)\end{array}$ & $\begin{array}{c}2.1 \\
(5.0)\end{array}$ & $\begin{array}{c}1.0 \\
(0.9)\end{array}$ \\
\hline $\operatorname{SEm}( \pm)$ & 0.55 & 0.25 & 0.30 & 0.20 & 0.14 & 0.21 & 0.26 \\
\hline LSD (0.05) & NS & 0.77 & 0.92 & 0.62 & 0.43 & 0.65 & 0.80 \\
\hline $\begin{array}{c}\mathrm{V}_{1}: \text { Calcutta } \\
\text { Single }\end{array}$ & $\begin{array}{c}10.1 \\
(118.6)\end{array}$ & $\begin{array}{c}10.1 \\
(104.2)\end{array}$ & $\begin{array}{c}9.4 \\
(90.5)\end{array}$ & $\begin{array}{c}9.6 \\
(93.8)\end{array}$ & $\begin{array}{c}7.7 \\
(67.9)\end{array}$ & $\begin{array}{c}5.8 \\
(43.4)\end{array}$ & $\begin{array}{c}5.2 \\
(37.1)\end{array}$ \\
\hline $\begin{array}{c}\mathrm{V}_{2}: \text { Calcutta } \\
\text { Double }\end{array}$ & $\begin{array}{c}9.5 \\
(95.2)\end{array}$ & $\begin{array}{c}9.2 \\
(86.4)\end{array}$ & $\begin{array}{c}9.0 \\
(83.4)\end{array}$ & $\begin{array}{c}8.8 \\
(79.1)\end{array}$ & $\begin{array}{c}6.9 \\
(54.2)\end{array}$ & $\begin{array}{c}5.3 \\
(39.1)\end{array}$ & $\begin{array}{c}4.7 \\
(29.9)\end{array}$ \\
\hline $\operatorname{SEm}( \pm)$ & 0.82 & 0.38 & 0.27 & 0.22 & 0.21 & 0.28 & 0.16 \\
\hline LSD (0.05) & NS & NS & NS & 0.68 & 0.65 & NS & 0.49 \\
\hline $\begin{array}{l}S_{1}:(30 \times 20) \\
\mathrm{cm}\end{array}$ & $\begin{array}{c}11.1 \\
(110.4)\end{array}$ & $\begin{array}{c}10.0 \\
(102.2)\end{array}$ & $\begin{array}{c}9.3 \\
(88.7)\end{array}$ & $\begin{array}{c}9.3 \\
(89.4)\end{array}$ & $\begin{array}{c}7.6 \\
(65.2)\end{array}$ & $\begin{array}{c}5.5 \\
(42.3)\end{array}$ & $\begin{array}{c}5.1 \\
(35.6)\end{array}$ \\
\hline $\begin{array}{l}S_{2}:(30 \times 25) \\
\mathrm{cm}\end{array}$ & $\begin{array}{c}8.4 \\
(103.4)\end{array}$ & $\begin{array}{c}9.3 \\
(88.4)\end{array}$ & $\begin{array}{c}9.1 \\
(85.1)\end{array}$ & $\begin{array}{c}9.0 \\
(83.5)\end{array}$ & $\begin{array}{c}7.0 \\
(56.8)\end{array}$ & $\begin{array}{c}5.6 \\
(40.2)\end{array}$ & $\begin{array}{c}4.7 \\
(31.4)\end{array}$ \\
\hline $\operatorname{SEm}( \pm)$ & 0.43 & 0.48 & 0.12 & 0.19 & 0.09 & 0.16 & 0.26 \\
\hline LSD (0.05) & 1.32 & NS & NS & NS & 0.28 & NS & NS \\
\hline
\end{tabular}


Table.13 Effect of management module, variety and spacing on population of $A$. besseyi in tuberose at different days after spray during 2015-16

\begin{tabular}{|c|c|c|c|c|c|c|c|}
\hline \multirow[t]{3}{*}{ Treatments } & \multicolumn{7}{|c|}{ Population of $A$. besseyi / $20 \mathrm{~g}$ of plant parts } \\
\hline & \multicolumn{7}{|c|}{ 2015-16 } \\
\hline & $\begin{array}{c}\text { Before } \\
\text { spray }\end{array}$ & $\begin{array}{c}7 \text { days } \\
{\text { after } 1^{\text {st }}}_{\text {spray }}\end{array}$ & $\begin{array}{c}14 \text { days } \\
\text { after 1st } \\
\text { spray }\end{array}$ & $\begin{array}{c}7 \text { days } \\
\text { after } 2^{\text {nd }} \\
\text { spray }\end{array}$ & $\begin{array}{l}14 \text { days } \\
\text { after } 2^{\text {nd }} \\
\text { spray }\end{array}$ & $\begin{array}{c}7 \text { days } \\
\text { after } 3^{\text {rd }} \\
\text { spray }\end{array}$ & $\begin{array}{c}14 \text { days } \\
\text { after } 3^{\text {rd }} \\
\text { spray }\end{array}$ \\
\hline $\mathbf{M}_{1}$ & $\begin{array}{c}10.5 \\
(115.8)\end{array}$ & $\begin{array}{c}10.8 \\
(121.3)\end{array}$ & $\begin{array}{c}10.5 \\
(113.0)\end{array}$ & $\begin{array}{c}10.0 \\
(100.8)\end{array}$ & $\begin{array}{c}10.4 \\
(110.5)\end{array}$ & $\begin{array}{c}10.1 \\
(102.5)\end{array}$ & $\begin{array}{c}8.8 \\
(77.8)\end{array}$ \\
\hline $\mathbf{M}_{2}$ & $\begin{array}{c}9.7 \\
(94.9)\end{array}$ & $\begin{array}{c}9.1 \\
(83.7)\end{array}$ & $\begin{array}{c}9.4 \\
(69.3)\end{array}$ & $\begin{array}{c}7.4 \\
(57.6)\end{array}$ & $\begin{array}{c}7.6 \\
(58.4)\end{array}$ & $\begin{array}{c}6.6 \\
(44.5)\end{array}$ & $\begin{array}{c}4.5 \\
(20.5)\end{array}$ \\
\hline $\mathbf{M}_{3}$ & $\begin{array}{c}8.2 \\
(68.5)\end{array}$ & $\begin{array}{c}8.6 \\
(75.1)\end{array}$ & $\begin{array}{c}7.2 \\
(51.9)\end{array}$ & $\begin{array}{c}4.8 \\
(21.7)\end{array}$ & $\begin{array}{c}4.1 \\
(17.4)\end{array}$ & $\begin{array}{c}2.7 \\
(8.1)\end{array}$ & $\begin{array}{c}1.4 \\
(2.1)\end{array}$ \\
\hline $\operatorname{SEm}( \pm)$ & 0.85 & 0.77 & 0.32 & 0.27 & 0.47 & 0.37 & 0.27 \\
\hline LSD (0.05) & NS & NS & 0.99 & 0.83 & 1.45 & 1.14 & 0.83 \\
\hline $\begin{array}{c}\mathrm{V}_{1}: \text { Calcutta } \\
\text { Single }\end{array}$ & $\begin{array}{c}9.9 \\
(101.9)\end{array}$ & $\begin{array}{c}10.0 \\
(102.5)\end{array}$ & $\begin{array}{c}9.5 \\
(94.2)\end{array}$ & $\begin{array}{c}7.7 \\
(64.6)\end{array}$ & $\begin{array}{c}7.9 \\
(69.5)\end{array}$ & $\begin{array}{c}6.8 \\
(55.5)\end{array}$ & $\begin{array}{c}5.2 \\
(37.1)\end{array}$ \\
\hline $\begin{array}{c}\mathrm{V}_{2}: \text { Calcutta } \\
\text { Double }\end{array}$ & $\begin{array}{c}9.0 \\
(84.2)\end{array}$ & $\begin{array}{c}9.0 \\
(84.2)\end{array}$ & $\begin{array}{c}8.5 \\
(74.3)\end{array}$ & $\begin{array}{c}7.1 \\
(54.6)\end{array}$ & $\begin{array}{c}6.8 \\
(54.7)\end{array}$ & $\begin{array}{c}6.1 \\
(47.9)\end{array}$ & $\begin{array}{c}4.7 \\
(29.9)\end{array}$ \\
\hline $\operatorname{SEm}( \pm)$ & 0.39 & 0.35 & 0.27 & 0.17 & 0.30 & 0.29 & 0.16 \\
\hline LSD (0.05) & NS & NS & 0.83 & 0.52 & 0.92 & NS & 0.49 \\
\hline $\begin{array}{l}\mathrm{S}_{1}:(30 \times 20) \\
\mathrm{cm}\end{array}$ & $\begin{array}{c}9.6 \\
(95.4)\end{array}$ & $\begin{array}{c}9.8 \\
(98.1)\end{array}$ & $\begin{array}{c}9.3 \\
(89.8)\end{array}$ & $\begin{array}{c}7.5 \\
(61.3)\end{array}$ & $\begin{array}{c}7.3 \\
(63.1)\end{array}$ & $\begin{array}{c}7.0 \\
(52.3)\end{array}$ & $\begin{array}{c}5.1 \\
(35.6)\end{array}$ \\
\hline $\begin{array}{l}\mathrm{S}_{2}:(30 \times 25) \\
\mathrm{cm}\end{array}$ & $\begin{array}{c}9.3 \\
(90.7)\end{array}$ & $\begin{array}{c}9.2 \\
(88.6)\end{array}$ & $\begin{array}{c}8.7 \\
(78.7)\end{array}$ & $\begin{array}{c}7.3 \\
(57.9)\end{array}$ & $\begin{array}{c}7.4 \\
(61.1)\end{array}$ & $\begin{array}{c}6.2 \\
(51.1)\end{array}$ & $\begin{array}{c}4.7 \\
(31.4)\end{array}$ \\
\hline $\operatorname{SEm}( \pm)$ & 0.63 & 0.17 & 0.14 & 0.16 & 0.45 & 0.40 & 0.26 \\
\hline LSD (0.05) & NS & NS & NS & NS & NS & NS & NS \\
\hline
\end{tabular}

Note: Data shown are $\sqrt{ } \mathrm{x}+0.5$ transformed values, figures in parentheses indicate original values, $\mathrm{M}_{1}=$ Untreated control, $\mathrm{M}_{2}=$ Farmer's practice module and $\mathrm{M}_{3}=$ Integrated pest management module 
Table.14 Effect of management module, variety and spacing on population of $A$. besseyi in tuberose at different days after spray (Pooled)

\begin{tabular}{|c|c|c|c|c|c|c|c|}
\hline \multirow[t]{3}{*}{ Treatments } & \multicolumn{7}{|c|}{ Population of $A$. besseyi / $20 \mathrm{~g}$ of plant parts } \\
\hline & \multicolumn{7}{|c|}{ Pooled } \\
\hline & $\begin{array}{c}\text { Before } \\
\text { spray }\end{array}$ & $\begin{array}{c}7 \text { days } \\
{\text { after } 1^{\text {st }}}_{\text {spray }}\end{array}$ & $\begin{array}{c}14 \text { days } \\
\text { after 1st } \\
\text { spray }\end{array}$ & $\begin{array}{c}7 \text { days } \\
\text { after } 2^{\text {nd }} \\
\text { spray }\end{array}$ & $\begin{array}{l}14 \text { days } \\
\text { after } 2^{\text {nd }} \\
\text { spray }\end{array}$ & $\begin{array}{c}7 \text { days } \\
\text { after } 3^{\text {rd }} \\
\text { spray }\end{array}$ & $\begin{array}{l}14 \text { days } \\
\text { after } 3^{\text {rd }} \\
\text { spray }\end{array}$ \\
\hline $\mathbf{M}_{1}$ & $\begin{array}{c}10.6 \\
(128.4)\end{array}$ & $\begin{array}{c}10.7 \\
(117.5)\end{array}$ & $\begin{array}{c}10.6 \\
(114.4)\end{array}$ & $\begin{array}{c}10.5 \\
(110.9)\end{array}$ & $\begin{array}{c}10.5 \\
(112.5)\end{array}$ & $\begin{array}{c}9.9 \\
(98.5)\end{array}$ & $\begin{array}{c}9.1 \\
(82.6)\end{array}$ \\
\hline $\mathbf{M}_{2}$ & $\begin{array}{c}9.8 \\
(97.6)\end{array}$ & $\begin{array}{c}9.1 \\
(85.9)\end{array}$ & $\begin{array}{c}9.0 \\
(70.5)\end{array}$ & $\begin{array}{c}8.1 \\
(68.2)\end{array}$ & $\begin{array}{c}7.4 \\
(55.5)\end{array}$ & $\begin{array}{c}5.7 \\
(34.4)\end{array}$ & $\begin{array}{c}5.8 \\
(34.9)\end{array}$ \\
\hline $\mathbf{M}_{3}$ & $\begin{array}{c}8.4 \\
(74.0)\end{array}$ & $\begin{array}{c}8.9 \\
(79.5)\end{array}$ & $\begin{array}{c}7.7 \\
(60.4)\end{array}$ & $\begin{array}{c}6.3 \\
(40.7)\end{array}$ & $\begin{array}{c}4.0 \\
(16.7)\end{array}$ & $\begin{array}{c}2.4 \\
(6.5)\end{array}$ & $\begin{array}{c}1.2 \\
(1.5)\end{array}$ \\
\hline SEm $( \pm)$ & 0.65 & 0.50 & 0.33 & 0.20 & 0.24 & 0.21 & 0.16 \\
\hline LSD (0.05) & NS & NS & 0.97 & 0.59 & 0.71 & 0.62 & 0.47 \\
\hline $\begin{array}{l}\mathrm{V}_{1} \text { : Calcutta } \\
\text { Single }\end{array}$ & $\begin{array}{c}10.0 \\
(110.3)\end{array}$ & $\begin{array}{c}10.0 \\
(103.4)\end{array}$ & $\begin{array}{c}9.5 \\
(92.4)\end{array}$ & $\begin{array}{c}8.7 \\
(79.2)\end{array}$ & $\begin{array}{c}7.8 \\
(68.7)\end{array}$ & $\begin{array}{c}6.3 \\
(49.5)\end{array}$ & $\begin{array}{c}5.5 \\
(43.1)\end{array}$ \\
\hline $\begin{array}{l}\mathrm{V}_{2}: \text { Calcutta } \\
\text { Double }\end{array}$ & $\begin{array}{c}9.2 \\
(89.7)\end{array}$ & $\begin{array}{c}9.1 \\
(85.3)\end{array}$ & $\begin{array}{c}8.8 \\
(78.8)\end{array}$ & $\begin{array}{c}8.0 \\
(66.8)\end{array}$ & $\begin{array}{c}6.9 \\
(54.4)\end{array}$ & $\begin{array}{c}5.7 \\
(43.5)\end{array}$ & $\begin{array}{c}5.1 \\
(36.2)\end{array}$ \\
\hline $\operatorname{SEm}( \pm)$ & 0.35 & 0.06 & 0.13 & 0.15 & 0.17 & 0.19 & 0.14 \\
\hline LSD (0.05) & NS & 0.18 & 0.38 & 0.44 & 0.50 & NS & NS \\
\hline$S_{1}:(30 \times 20) \mathrm{cm}$ & $\begin{array}{c}10.3 \\
(102.9)\end{array}$ & $\begin{array}{c}9.9 \\
(100.2)\end{array}$ & $\begin{array}{c}9.3 \\
(89.3)\end{array}$ & $\begin{array}{c}8.4 \\
(75.4)\end{array}$ & $\begin{array}{c}7.5 \\
(64.2)\end{array}$ & $\begin{array}{c}6.1 \\
(47.3)\end{array}$ & $\begin{array}{c}5.5 \\
(41.3)\end{array}$ \\
\hline $\mathrm{S}_{2}:(30 \times 25) \mathrm{cm}$ & $\begin{array}{c}8.9 \\
(97.1)\end{array}$ & $\begin{array}{c}9.3 \\
(88.5)\end{array}$ & $\begin{array}{c}8.9 \\
(81.9)\end{array}$ & $\begin{array}{c}8.2 \\
(70.7)\end{array}$ & $\begin{array}{c}7.2 \\
(59.0)\end{array}$ & $\begin{array}{c}5.9 \\
(45.7)\end{array}$ & $\begin{array}{c}5.2 \\
(38.0)\end{array}$ \\
\hline SEm $( \pm)$ & 0.55 & 0.34 & 0.28 & 0.11 & 0.18 & 0.20 & 0.15 \\
\hline LSD (0.05) & NS & NS & NS & NS & NS & NS & NS \\
\hline
\end{tabular}

Note: Data shown are $\sqrt{\mathrm{x}+0.5}$ transformed values, figures in parentheses indicate original values, $\mathrm{M}_{1}=$ Untreated control, $\mathrm{M}_{2}=$ Farmer's practice module and $\mathrm{M}_{3}=$ Integrated pest management module.

Table.15 Comparative economics of adopted foliar nematode management modules in tuberose over the growing period of 2014-16

\begin{tabular}{|c|c|c|c|c|c|c|c|c|c|c|c|}
\hline \multirow[t]{3}{*}{ Module } & \multirow{3}{*}{$\begin{array}{l}\text { Total } \\
\text { yield of } \\
\text { loose } \\
\text { flowers in } \\
\text { two years } \\
\text { (q/ha) }\end{array}$} & Yield of & Gain in flower & $\begin{array}{c}\text { Gain in bulb } \\
\text { yield }\end{array}$ & $\begin{array}{c}\begin{array}{c}\text { Value of } \\
\text { additional }\end{array} \\
\end{array}$ & $\begin{array}{c}\text { Total chemical } \\
\text { used per }\end{array}$ & \multirow{2}{*}{\multicolumn{2}{|c|}{$\begin{array}{c}\begin{array}{c}\text { Cost of treatment in } \\
\text { two }\end{array} \\
\text { years (Rs./ha) }\end{array}$}} & \multirow{3}{*}{ 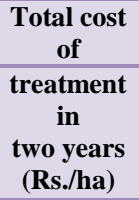 } & \multirow{3}{*}{\begin{tabular}{|l|}
$\begin{array}{l}\text { Net } \\
\text { gain }\end{array}$ \\
(Rs./h \\
a) \\
\end{tabular}} & \multirow{3}{*}{$\begin{array}{c}\text { Incremental } \\
\text { Cost: } \\
\text { Benefit }\end{array}$} \\
\hline & & \multicolumn{2}{|c|}{$\begin{array}{c}\text { bulb after yield over } \\
\text { control }\end{array}$} & \multirow[t]{2}{*}{$\begin{array}{l}\text { over control } \\
\quad(q / h a)\end{array}$} & \multirow{2}{*}{$\begin{array}{l}\text { flowers } \\
\text { and bulb } \\
\text { yield } \\
\text { (Rs./ha) }\end{array}$} & \multirow[t]{2}{*}{ ha in two years } & & & & & \\
\hline & & $\begin{array}{c}2^{\text {nd }} \text { year } \\
\text { (q/ha) }\end{array}$ & $\begin{array}{c}\text { in two years } \\
(\mathrm{q} / \mathrm{ha})\end{array}$ & & & & Chemical & Labour & & & \\
\hline $\begin{array}{l}\text { Farmers' } \\
\text { practice } \\
\text { module }\end{array}$ & 154.30 & 334.20 & 49.90 & 113.10 & 111110.00 & $\begin{array}{c}\text { Monocrotophos } \\
36 \\
\text { SL } 17 \text { litres }\end{array}$ & 5950.00 & 1944.00 & 7894.00 & $\begin{array}{l}103216 \\
.00\end{array}$ & 1:14.08 \\
\hline $\begin{array}{l}\text { IPM } \\
\text { module }\end{array}$ & 218.20 & 454.70 & 113.80 & 233.60 & 250960.00 & $\begin{array}{c}\text { Cartap } \\
\text { hydrochloride } \\
50 \text { SP } 13 \mathrm{~kg}+ \\
\text { Chlorfenapyr } 10 \\
\text { SC } \\
2 \text { litres + Active } \\
801 \text { litre }\end{array}$ & 12608.00 & 1458.00 & 14066.00 & $\begin{array}{l}236894 \\
.00\end{array}$ & $1: 17.84$ \\
\hline $\begin{array}{l}\text { Untreated } \\
\text { control }\end{array}$ & 104.4 & 221.1 & - & - & - & & - & - & - & - & - \\
\hline
\end{tabular}

Note: Market price of monocrotophos 36SL @ Rs.350/litre, cartap hydrochloride 50 SP @ Rs.650/kg, chlorfenapyr 10 SC @ Rs. 900/kg, tuberose flowers (loose) @ Rs. 2000/q, tuberose bulbs @ Rs. 100/q, labour wages @ Rs. 243/manday. 
Nematode management module may not influence the spike yield significantly (based on pooled result) but the flower yield ( $\mathrm{kg} / \mathrm{plot}$ and $\mathrm{q} / \mathrm{ha})$ which was worked out based on spike yield was varied significantly due to adoption of the integrated foliar nematode management tactics as evident from the result (Table 7 and 8).

The variety Calcutta Single yielded significantly maximum number of spikes in both the years which was 106.5 and 96.0/plot and 4,43,750 and 4,00,000/ha during 2014-15 and 2015-16, respectively (Table 10 and 11). Pooled data also showed similar outcome.

Planting of bulbs in wide spacing $(30 \mathrm{~cm} \times 25 \mathrm{~cm})$ yielded significantly maximum number of spikes i.e. $3,10,880$ and $2,71,065 /$ ha for the $1^{\text {st }}$ and $2^{\text {nd }}$ year, respectively (Table 11). Though the effect of spacing on spike yield was non-significant considering pooled data but the trend was similar to that of individual year-wise observation (Table 10 and 11).

Effect of management module, variety and spacing on population of $A$. besseyi in tuberose at different days after spray during 2014-15 and 2015-16

Effect of foliar nematode management module, variety and spacing on population of $A$. besseyi in tuberose was studied at different days after spray during 2014-15 and 2015-16. Total number of foliar spray of nematicides was three and four respectively for the IPM module and famers' practice, respectively in each year. In farmers' practice, spray was initiated at the commencement of $A$. besseyi infestation; while, in IPM module spray was initiated at the time of floral spike emergence. For the ease of comparison of performance of the nematicidal spray included in the management module, nematode population (number $/ 20 \mathrm{~g}$ of plant parts) data at regular interval after three round of spray has been presented in tabular form below (Table 12-14). Significant difference in population (number/20g plant parts) of $A$. besseyi between management modules at 7 and 14 days after each round of spray was observed in the $1^{\text {st }}$ year (Table 12). All time except 7 days after $1^{\text {st }}$ round of spray, the nematode population varied significantly between management modules as found in the second year and pooled result (Table 13 and 14). Population of $A$. besseyi in tuberose before spray didn't vary significantly (Table 12-14). Population of $A$. besseyi / $20 \mathrm{~g}$ of plant parts at seven days after $1^{\text {st }}$ round of spray with cartap hydrochloride 50 SP @ 500g a.i. /ha + Active 80 (adjuvant) @ 5ml/15 litres of water in $\mathrm{M}_{3}$ (IPM module) was found statistically at par with the spray of monocrotophos 36 SL @ $360 \mathrm{~g}$ a.i./ha in $\mathrm{M}_{2}$ (farmers' practice module) in each year (Table 12 and 13).

The variation in foliar nematode population between $\mathrm{M}_{2}$ and $\mathrm{M}_{3}$ was significant at 14 days after $1^{\text {st }}$ round of spray with the least being, $51.9 / 20 \mathrm{~g}$ plant parts in $\mathrm{M}_{3}$ in the $2^{\text {nd }}$ year (Table 13). In the first year, significant variation in foliar nematode population between $\mathrm{M}_{2}$ and $\mathrm{M}_{3}$ was observed first at 7 days after second round of spray of nematicides, the least was 59.8/20g plant parts recorded in $\mathrm{M}_{3}$ which involves spray of chlorfenapyr10 SC @100g a.i. /ha + Active 80 (adjuvant)@5ml/15 litres of water. Thereafter, a significant gradual decline in the nematode population at regular interval was observed due to adoption of nematicidal sprays in the 'famers' practice' and 'IPM' module. Least nematode population was noted at 14 days after third round of spray with cartap hydrochloride 50 SP @ 500g a.i. /ha + Active 80 (adjuvant) @ 5ml/15 litres of water i.e. $\mathrm{M}_{3}$ (Table 12-14).

Out of two varieties of tuberose, Calcutta Single had more population of foliar nematode than the Calcutta Double in general. Based on pooled analysis of two years data, significantly low population of $A$. besseyi was recorded in the Calcutta Double variety all time except at 7 days after $3^{\text {rd }}$ round of spray. Population of nematodes between varieties didn't vary before spray of nematicides.

Population of nematodes between spacings didn't vary significantly due to spray of nematicides (pooled, Table 14) but wide spaced crop had comparatively lesser population than the narrow spaced crop and this probably helped the widespaced crop to achieve better growth. The better plant growth contributed a better flower yield in wide-spaced crop (Table 8). 
Population of $A$. besseyi per $20 \mathrm{~g}$ of plant parts before spray was also least with the IPM module $\left(\mathrm{M}_{3}\right)$ where overnight pre-soaking of bulbs followed by dipping in cartap hydrochloride 50 SP @ 1000ppm for 4 hrs before planting was practiced. This was followed by the farmers' practice module $\left(\mathrm{M}_{2}\right)$ where overnight pre-soaking of bulbs followed by dipping in monocrotophos 36 SL @ 720ppm for 4 hrs before planting was adopted. That is why nematode population before spray was lesser in the modules having bulb treatment than the untreated one. Even after three rounds of spray, much decline in nematode population was not noticed in the farmers' practice module. So, a fourth round of spray with monocrotophos 36 SL @ 360 g a.i./ha was given here.

Bulb treatment with cartap hydrochloride $50 \mathrm{SP}$ @ 1000ppm was found more helpful than the bulb treatment with monocrotophos 36 SL @ 720ppm to keep the nematode population low (Table 14).

Comparative economics of adopted foliar nematode management modules in tuberose over the growing period of 2014-16

The IPM module was much better in increasing the production of flowers and bulbs of tuberose and securing net gain almost double to that of farmers' practice module. The incremental cost benefit ratio was found more in IPM module than the farmers' practice module. The maximum return per rupee investment for managing $A$. besseyi in tuberose was observed with the IPM module being, Rs. 17.84 (Table 15).

Despite more treatment cost high incremental benefit of treatment was obtained in the IPM module of foliar nematode management than the farmers' practice module. The maximization of flower and bulb yields due to adoption of foliar nematode management treatments was observed in the IPM module. That possibly helped to achieve maximum incremental benefit in the IPM module. Farmers' practice module also achieved benefit due to adoption of nematode management protocol but was inferior to that of IPM module. Significant cumulative effect of foliar nematode management module, spacing and variety on the spike length, rachis length, number of nodes/spike and days to 1 st and $100 \%$ blooming was not observed in the study. Individually the management module and the variety had shown positive impact on the spike length, rachis length and the number of nodes/spike of tuberose crop. Only variety had significant effect on the days to 1 st and $100 \%$ blooming of

tuberose. Spacings on the other hand revealed non-significant influence on the spike length, rachis length, number of nodes/spike, days to $1 \mathrm{st}$ and $100 \%$ blooming and number of flowers/spike.

In the present study, spike length, rachis length, number of nodes/spike and flower yield (number and weight basis) were usually observed maximum when widely spaced crop

was raised adopting IPM module. The effect was not statistically significant. Widely grown plants harbour less foliar nematode population and also availability of more nutrients to each plant made the spacing superior over narrow-spaced crop.

Kumar et al., (2002) observed increased leaf number, flower production, spike and rachis length from wider spaced $(30 \mathrm{~cm} \times 30 \mathrm{~cm})$ bulbs of tuberose cv. Single. Improved flower production and heavier individual florets was observed with wider spaced $(30 \mathrm{~cm} \times 30 \mathrm{~cm})$ tuberose plants (Khobragade et al., 1997; Singh and Sangama, 2000; Kumar et al., 2002). The IPM module of foliar nematode management comprising of bulb treatment, field sanitation and prophylactic spraying with nematicides in the present experiment kept the $A$. besseyi population (as judged by the population per $20 \mathrm{~g}$ of plant parts at different time interval after spray) significantly lower than the farmers' practice module and untreated control. Hence, adoption of IPM module possibly made the crop to achieve good growth and development as judged by the several attributes (spike length, rachis length, number of nodes/spike, flower yields etc.) measured during the course of experimentation.

In conclusion, the IPM module of foliar nematode management comprising of bulb treatment, field sanitation and prophylactic spraying with nematicides in the present experiment kept the $A$. besseyi population significantly lower than the farmers' practice module and untreated control. Hence, adoption of IPM module possibly made 
the crop to achieve good growth and development as judged by the several attributes (spike length, rachis length, number of nodes/spike, flower yields etc.) measured during the course of experimentation. The maximum return per rupee investment for managing $\mathrm{A}$. besseyi in tuberose was observed with the IPM module being, Rs. 17.84. To enhance the flower yield of tuberose, Calcutta Single cultivar can be planted in $30 \mathrm{~cm} \times$ $25 \mathrm{~cm}$ spacing adopting IPM module of foliar nematode management. Validation and promotion of developed IPM module of foliar nematode management for the farmers raising tuberose in the Gangetic plains of West Bengal need to be done.

\section{References}

Ahmed, M.J., Bashir, T., Yaqoob, A., Jilani, M.S. and Saeed, M. 2010. Effect of plant spacing on vegetative and reproductive growth of Gladiolus cultivars. Sarhad Journal of Agriculture, 26: 539-543.

Desai, N., Mamatha, B. and Patil, R. B. 2016. Effect of spacing and fertilizer levels on flowering and concrete yield in tuberose (Polianthes tuberosa L.) cv. Shringar. Journal of Farm Science, 29(2): 290-293.

Gomez, K.A. and Gomez, A.A. 1984. Statistical Procedures for Agricultural Research, $2^{\text {nd }}$ Edition. A Willey Inter-science Publications, John Willey and Sons, pp. 680.

Khan, M.R. and Pal, A.K. 2001. Plant parasitic nematodes associated with tuberose (Polianthes tuberosa L.) in West Bengal. Annals of Plant Protection Sciences, 9: 357-359.

Khobragade, R.I., Damke. M.M., Jadhao. B.J. and Hedau C.V. 1997. Effect of planting time and spacing on growth, flowering and bulb production of tuberose cv. 'Single'. $P K V$ Research Journal, 21: 44-47.

Kumar, R., Gobind, S. and Yadav, D. S. 2002.
Studies on $\mathrm{N}$ and $\mathrm{P}$ requirement of tuberose (Polianthes tuberosa Linn.) cv. Single in hilly soils. Haryana Journal of Horticulture Science, 31(1/2): 52-54.

Malam, V.R., Singh, S.P., Ahlawat T.R., Mathukia, R.K. and Jat, G. 2010. Effect of spacing and crop duration on growth, flowering and bulb production in tuberose (Polianthes tuberosa L.) cv. Double. Journal of Horticultural Sciences, 5(2): 134-137.

Mishra, H., Singh, A.K. and Singh, O.P. 2000. Effect of bulb size and spacing on growth and flowering behaviour of tuberose (Polianthes tuberosa L.). Advance Plant Science, 13(2): 115-125.

Mohanty, C.R., Mishra, M. and Mohapatra, A. 2002. In: Floriculture Research Trend in India, Misra, R.L. and Misra, S. (eds.). Proceedings of National Symposium on Indian Floriculture in the new Millennium, pp. 340-342.

Pathak, B. and Khan, M.R. 2009. Yield loss potential of foliar nematode, Aphelenchoides besseyi in tuberose. Indian Journal of Nematolology, 39(1): 38-40.

Roy, K., Sarkar, H.K. and Pramanik, A. 2010. Morpho-anatomical changes in Gladiolus due to infection by Aphelenchoides besseyi from West Bengal, India. Floriculture and Ornamental Biotechnology, 4: 96-98.

Roy, K., Sarkar, H.K. and Pramanik, A. 2011. Symptoms and morpho-anatomical changes in leaves of Polianthes tuberosa with infection of Aphelenchoides besseyi. Annals of Plant Protection Sciences, 19(1): 249-250.

Sheoran, S., Dudi, O.P., Beniwal, B.S. and Dalal, R.P.S. 2015. Effect of nitrogen and Spacing on flowering and yield of tuberose (Polianthes tuberose L.) cv. Prajwal. Annals of Biology, 31(2): 270-274.

Singh, K.P. and Sangama. 2000. Effect of fertilizer on growth and flowering of tuberose (Polianthes tuberosa). Journal of Applied Horticulture, 2(1): 54-55.

\section{How to cite this article:}

Shimpy Sarkar and Kusal Roy. 2019. Integrated Management of Aphelenchoides besseyi Infesting Tuberose in Gangetic Plains of West Bengal. Int.J.Curr.Microbiol.App.Sci. 8(02): 3299-3315. doi: https://doi.org/10.20546/ijcmas.2019.802.385 